\title{
Research Article \\ Research on Fault Diagnosis Based on Singular Value Decomposition and Fuzzy Neural Network
}

\author{
Jingbo Gai and Yifan $\mathrm{Hu}$ \\ College of Aerospace and Civil Engineering, Harbin Engineering University, Harbin, Heilongjiang 150001, China \\ Correspondence should be addressed to Yifan Hu; 2012027205g@hrbeu.edu.cn
}

Received 10 January 2018; Accepted 7 March 2018; Published 8 April 2018

Academic Editor: Giosuè Boscato

Copyright ( 2018 Jingbo Gai and Yifan Hu. This is an open access article distributed under the Creative Commons Attribution License, which permits unrestricted use, distribution, and reproduction in any medium, provided the original work is properly cited.

\begin{abstract}
A method based on singular value decomposition (SVD) and fuzzy neural network (FNN) was proposed to extract and diagnose the fault features of diesel engine crankshaft bearings efficiently and accurately. Firstly, vibration signals of crankshaft bearings in known state under the same working condition were decomposed by EMD to obtain the modal components containing faultfeature information. Then, the singular values of modal components which include the main fault features were used as the initial vector matrix, where the eigenvectors were decomposed to form a fault characteristic matrix. At last, the fault features matrix was trained by the fuzzy neural network, in order to realize the diagnosis and identification of the crankshaft bearings in different states in the form of numerical values. The experiment showed that the numerical identification of the fuzzy neural network based on the singular value had high fault diagnosis accuracy and stability. This method can also reflect the gradual change of the crankshaft bearings' fault to some extent, so it has the desired reliability and value.
\end{abstract}

\section{Introduction}

Crankshaft bearing is an important part of the diesel engine with a high failure and abrasion rate. If we can extract faultfeature information from the vibration signals effectively and identify the operating state of the crankshaft bearing and then repair or replace it in time, it will be of great significance for the safety and economy of the diesel engine [1].

If we want to classify different fault states exactly, it is necessary to extract accurate signal features and identify these features accurately. Many experts have done a great deal of research on this. Yang et al. [2] decomposed nonstationary vibration signals into a series of intrinsic mode functions with empirical mode decomposition and input their energy into the neural network for fault diagnosis, and this method achieved good results; Xia et al. [3] proposed a method of fault diagnosis based on the combination of empirical mode decomposition and AR spectroscopy; Si et al. [4] used wavelet packet to denoise the vibration signal, extracted the eigenvalue of the fault signal, and used the fuzzy neural network to diagnose and recognize the fault states. With reference to the modal aliasing of EMD, Zhang et al. [5] extracted engine crankshaft fault features by integrated empirical mode decomposition, which can identify different fault states efficiently. However, the above extraction methods of fault features still need to be further discussed in terms of accuracy and stability. Singular values are the inherent features of the matrix. When the matrix elements change slightly, the singular values of the matrix change very little. At the same time, singular values have scale invariance and rotation invariance. Therefore, based on empirical mode decomposition, singular value decomposition can effectively solve the above-mentioned problem. Guo et al. [6] constructed the time-frequency matrix of the vibration signal by Hilbert-Huang transform, and the singular value of the matrix was regarded as the feature vector, and then they used FCM to classify the fault states of the distribution switch. Zhou et al. [7] proposed a fault diagnosis method of rolling bearing based on EMD-SVD and FCM. Although the extraction of fault features was effectively improved by singular value decomposition, it is still worth a further discussion on the accuracy and stability of diagnosis and recognition. As an important intelligent information processing method, fuzzy neural network has strong self-learning and data direct 
processing ability, so it can express the structure of the result clearly. Therefore, the fuzzy neural network can be used to classify the features of different faults in the form of numerical value; this method can also reflect the gradient of fault to some extent.

This paper combined the advantages of the abovementioned method in the extraction and recognition of fault features for the first time; a new fault diagnosis method based on EMD-SVD and fuzzy neural network was proposed. It was proved by experiment that this method can effectively compensate the shortage of previous research, and it had high accuracy and stability of fault diagnosis.

\section{The Introduction of EMD-SVD}

2.1. Empirical Mode Decomposition (EMD). Empirical mode decomposition is a smoothing process, which can decompose a complex, nonstationary signal into a number of intrinsic mode functions, and we abbreviate it as IMF [8]. The instantaneous frequency of any point in the IMF is meaningful. At any given moment, the signal can contain several IMF components. Each IMF's frequency and composition are different and the decomposition process is self-adaptive, so EMD is very suitable for dealing with nonstationary bearing vibration signals [9]. In the process of empirical mode decomposition, each IMF component must satisfy the following two conditions: firstly, the number of the extreme points and the zero points should be equal to or not more than one; secondly, the mean of the maximum and minimum of extreme points is zero at any point in the signal curve [10]. Since most of the nonstationary signals do not satisfy the IMF conditions directly, Huang made the following assumptions [11]: any complex signal is composed of IMFS which are independent of each other and each IMF component can be linear or nonlinear. For the signal $X(t)$, its empirical mode decomposition equation can be expressed as

$$
X(t)=\sum_{i=1}^{n} C_{i}(t)+r(t) .
$$

In the equation, $C_{i}(t)$ means the IMF components, $r(t)$ means the residual component, and it represents the average trend of the signal.

However, vibration signals of diesel engine crankshaft bearings often contain noises or intermittent signals, and this makes empirical mode decomposition exhibit a shortage of modal aliasing, so the extraction of signal features becomes unstable and inaccurate [12]. Singular value decomposition can effectively reduce the interference of noise components and intermittent signals, so singular value decomposition based on empirical mode decomposition can effectively extract the stable fault features. In the process of solving practical problems, we often focus on the effective IMF components, which contain the main fault-feature information. Therefore, selecting the effective IMF components is also a key step of fault-feature extraction in this paper.

2.2. Singular Value Decomposition (SVD). Singular value decomposition (SVD) is an important orthogonalization method of matrix decomposition in linear algebra. For a linear correlation matrix of rows or columns, it can be transformed into a linearly independent one by multiplying an orthogonal matrix on its left and right side, respectively. For a real matrix, $A_{m \times n}$, whose rank is $r$, if there exist two orthonormal matrices, $U$ and $W$, and another diagonal matrix, $D$, they satisfy the following equation:

$$
\begin{aligned}
& A_{m \times n}=U_{m \times m} D_{m \times n} W_{n \times n}{ }^{T}=\sum_{i}^{r} \delta_{i} u_{i} w_{i}^{T} \\
& U^{T} U=E_{m \times m} \quad W^{T} W=E_{n \times n} .
\end{aligned}
$$

Equation (2) is called the singular value decomposition of the real matrix $A_{m \times n}$.

In this equation, $U_{m \times m}=\left[u_{1}, u_{2}, \ldots, u_{m}\right], D_{m \times n}=$ $\left[\begin{array}{cc}\Delta_{r \times r} & 0 \\ 0 & 0\end{array}\right], \Delta_{r \times r}=\operatorname{diag}\left(\delta_{1}, \delta_{2}, \ldots, \delta_{r}\right), W_{n \times n}=\left[w_{1}, w_{2}, \ldots, w_{n}\right]$, $r=\min (m, n)$, and $\delta_{i}(i=1,2, \ldots, r)$ are the singular values of the real matrix $A_{m \times n} . \delta_{i}=\sqrt{\lambda_{i}}, \lambda_{1} \geq \lambda_{2} \geq \cdots \geq \lambda_{r} \geq 0$, are the eigenvalues of $A^{T} A$. Under the restrictions of $\lambda_{1} \geq \lambda_{2} \geq$ $\cdots \geq \lambda_{r} \geq 0$, the singular value of the matrix $\left(\delta_{1}, \delta_{2}, \ldots, \delta_{r}\right)$ is unique [10]. The singular value has the following two features: (1) the singular values of matrices have a better stability and (2) the singular values also have both proportion invariance and rotation invariance. Therefore, singular values can reflect the features of eigenvectors very well. In the process of constructing the real matrix $A$, the time delay embedding technique is usually used to reconstruct the phase space of one-dimensional time series. However, there is no clear theoretical guidance on how to determine the embedding dimension and the delay constant. For this problem, this paper combined EMD and SVD and formed the initial eigenvector matrix automatically by the IMF components with EMD. This combined EMD and SVD method can avoid the arbitrary choice of embedding dimensions and delay constants [10].

\section{Fuzzy Neural Network}

Fuzzy neural network is an important intelligent information processing method, which combines the advantages of fuzzy logic and neural networks well. Therefore, the fuzzy neural network algorithm not only has a strong self-learning ability to deal with data directly but also has a strong ability of structural knowledge expression. Figure 1 shows the general structure of the fuzzy neural network.

In the fuzzy neural network, the function of the first layer is to transfer the input signal to the next layer without any change. Each of its nodes corresponds to an input constant. The number of nodes in the input layer is determined by the input signal. In this paper, the number of input nodes is consistent with the number of key IMF components. The second layer is the quantized input layer; its function is to fuzzify the input variables. The third layer is the hidden layer; its function is to realize the mapping between the fuzzy values of the input variables and the output variables. The fourth layer is the quantized output layer; its output result is the fuzzification value. The fifth layer is the weighted output layer; it can make sure of the clarity of the output results. 


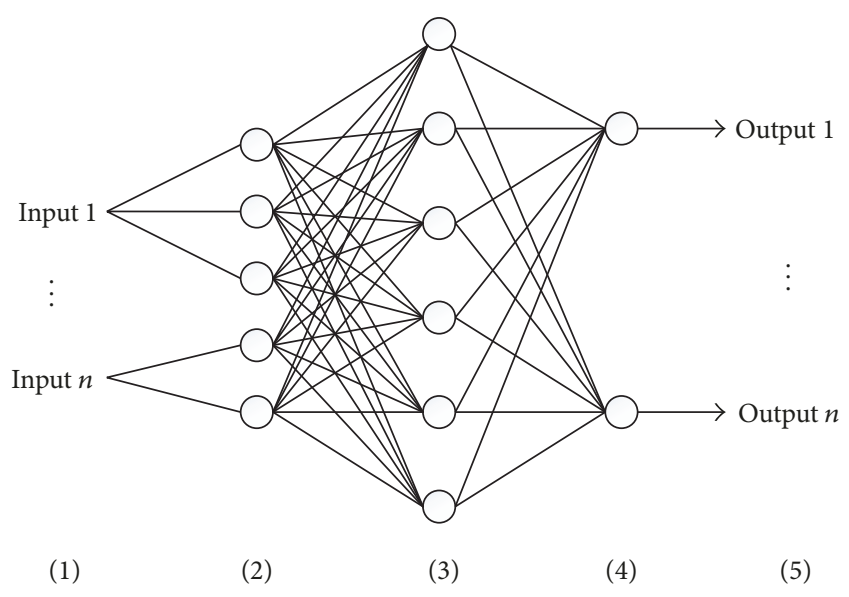

FIGURE 1: Structure diagram of the fuzzy neural network.

Network learning is a process of updating network connection parameters constantly, and it ultimately makes the network achieve optimal performance [4]. The learning error is calculated according to the actual output value and the target value, and then the condition parameter is adjusted by the back-propagation error. The specific adjustment process is as follows.

(1) Network initialization: determine the number of input nodes, hidden nodes, and output nodes of the network; initialize parameters of fuzzy neural network and fuzzy membership degree at the same time.

(2) The training of fuzzy neural network: input the sample $X_{n}(n=1,2, \ldots, n)$ and the label $Y_{n}(n=1,2, \ldots, n)$, and then set the hyperparameter (such as the number of iterations and the network learning rate).

(3) Start iteration: adjust the network connection parameters constantly until the actual output of the network is consistent with the ideal one; then, the training is over.

(4) Classify the samples with the trained fuzzy neural network.

\section{Fault Diagnosis Based on EMD-SVD and Fuzzy Neural Network}

The fault diagnosis method based on EMD-SVD and fuzzy neural network is shown in Figure 2.

The specific diagnostic steps are as follows.

Empirical mode decomposition: first, pick out the vibration signals $X(t)$ of crankshaft bearings in normal state, slight wear, and severe wear under the same condition and decompose the signals with EMD; we can obtain several IMF components with different feature scales $c_{1}, c_{2}, \ldots, c_{n}$. Then, form the initial eigenvector matrix $A$ with the IMF components, $A=\left[c_{1}, c_{2}, \ldots, c_{n}\right]^{T}$.

Singular value decomposition: decompose the key IMF components in the initial eigenvector matrix with SVD and then obtain the singular values, $\delta=\left[\delta_{1}, \delta_{2}, \ldots, \delta_{n}\right], \delta_{1} \geq$ $\delta_{2} \geq \cdots \geq \delta_{n}$. And the singular values are used as the final fault-feature vector.

Fault diagnosis of fuzzy neural network: the fault-feature matrix was formed by the singular values of three kinds of
TABLE 1: The relevant parameters of the test and diesel engine.

\begin{tabular}{lcc}
\hline Rated power & Speed & Sampling frequency \\
\hline $3990 \mathrm{KW}$ & $1500 \mathrm{r} / \mathrm{min}$ & $5.12 \mathrm{kHz}$ \\
\hline
\end{tabular}

bearings' states under the same working condition. And the singular values are also used as an input training sample of fuzzy neural network. At the same time, three groups of signals are picked out from each state, and then we verify the identification of the fuzzy neural network. The specific diagnostic methods are as follows: after learning and training, each fault state will be trained as a numerical value; we can distinguish different fault states by the numerical values, and the approximation between the output results and the numerical indexes can effectively reflect the gradient of the fault.

\section{Experimental Analysis}

DH5922N dynamic signal tester was used with appropriate IEPE piezoelectric acceleration sensors to measure the vibration of diesel engine crankshaft bearing in this experiment. The acceleration sensor is placed on the bearing seat. Connecting the tester with a computer, we can monitor and collect the vibration signals of crankshaft bearings and distinguish the vibration features of crankshaft bearings in normal state, slight wear, and severe wear. The relevant parameters of the test and diesel engine are shown in Table 1 . When the diesel engine worked at the rated power, the time-domain vibration signals of crankshaft bearing collected in different states are shown in Figure 3.

5.1. The Extraction of Fault Features. Decompose the vibration signals in the three above-mentioned states (normal state, slightly worn state, and severely worn state) with EMD (take a group of vibration signals in normal state as an example). The time and frequency-domain signals after empirical mode decomposition are shown in Figure 4.

Figure 4(a) shows the time-domain diagram of the first 8-order IMF obtained after EMD of the above vibration signal. The abscissa axis indicates the temporal variation, and the ordinate indicates the amplitude. IMF1-IMF8 represent the relationship between the amplitude and the sampling time of each intrinsic mode function. Figure 4(b) shows the frequency-domain diagram of IMF1-IMF8, the abscissa axis indicates the frequency, and the ordinate indicates the amplitude. The spectrum shows the frequency range in which different intrinsic mode functions concentrate. From the first eight IMF components and their spectrums, it is easily found that the energy of the signals was almost occupied by the first five IMF components. Therefore, the first five IMF components can be considered as the key IMF components, and they are used to generate the initial vector matrix (vibration signals of bearings in slightly worn state and severely worn state were treated in the same way; each state will be taken in five groups of signals).

Decompose the initial vector matrix with SVD. The result of singular value decomposition in different states of 


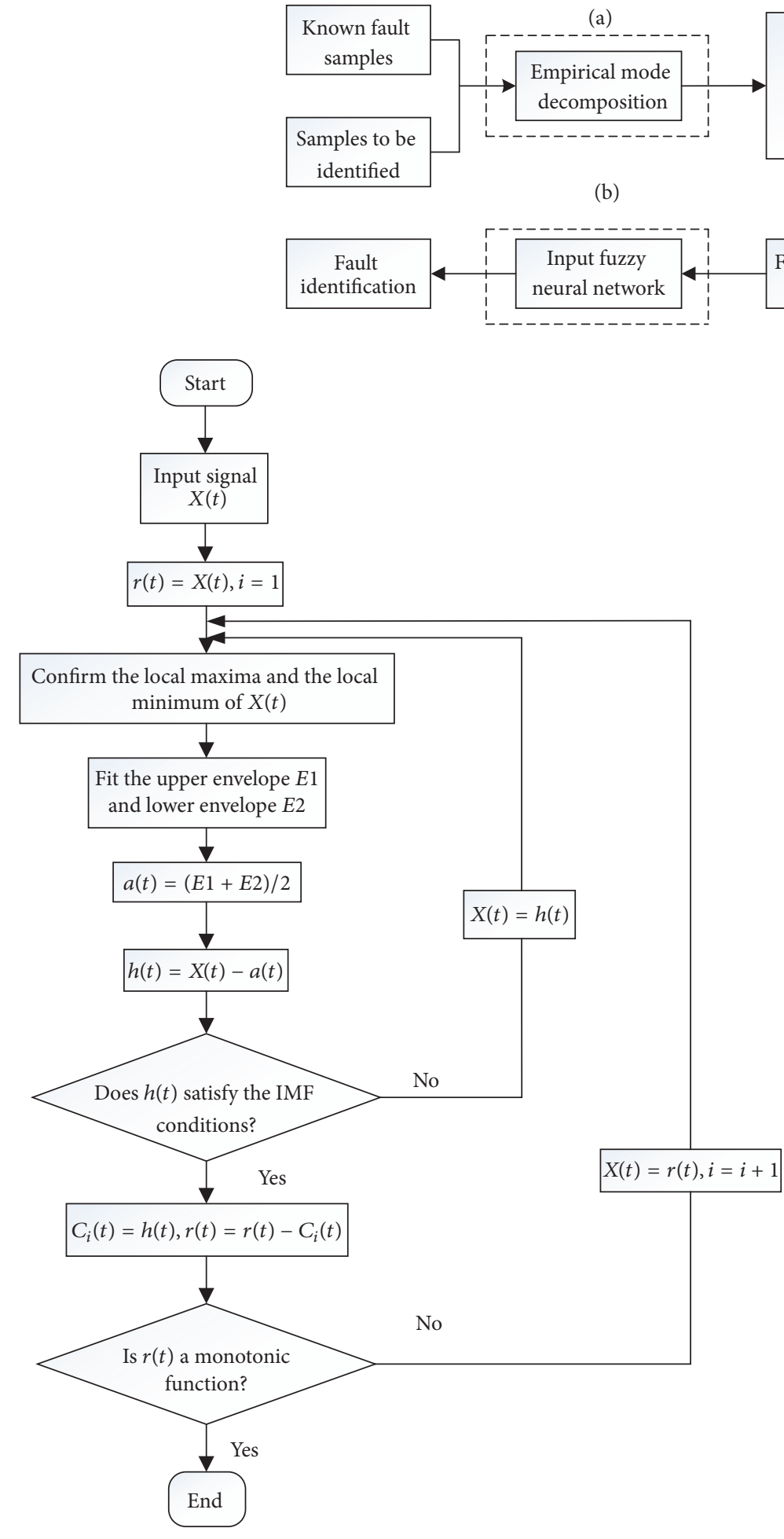

(a)

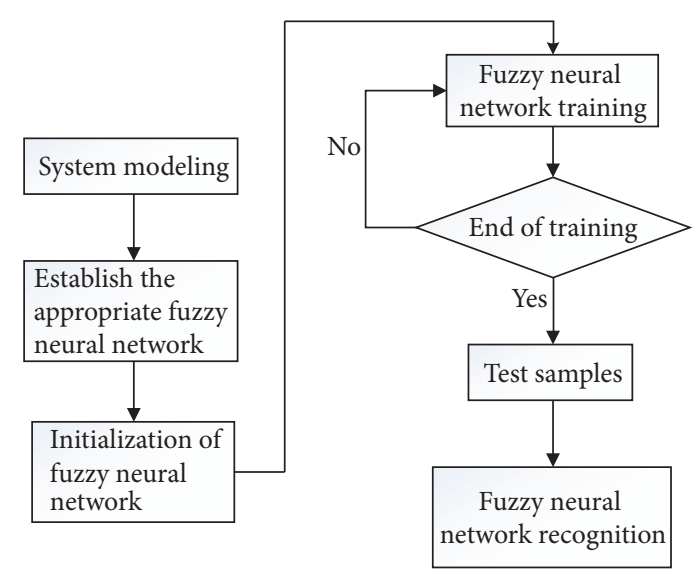

(b)

FIGURE 2: Fault diagnosis method based on EMD-SVD and fuzzy neural network. 
TABLE 2: Singular value decomposition results of vibration signals in different states of crankshaft bearing.

\begin{tabular}{|c|c|c|c|c|c|c|}
\hline Wear degree of crankshaft bearing & Signal & $\delta_{1}$ & $\delta_{2}$ & $\delta_{3}$ & $\delta_{4}$ & $\delta_{5}$ \\
\hline \multirow{5}{*}{ Normal state } & Group 1 & 102.3797 & 75.3013 & 54.9141 & 26.9316 & 14.4099 \\
\hline & Group 2 & 106.5650 & 78.3713 & 51.4455 & 20.2149 & 11.7647 \\
\hline & Group 3 & 107.3573 & 78.9566 & 46.7620 & 25.6204 & 12.5991 \\
\hline & Group 4 & 110.3927 & 75.6752 & 46.3543 & 25.3347 & 15.1932 \\
\hline & Group 5 & 108.7006 & 74.8050 & 51.1752 & 24.5572 & 14.2192 \\
\hline \multirow{5}{*}{ Slight wear } & Group 1 & 217.9721 & 64.7321 & 37.0377 & 27.6948 & 15.9726 \\
\hline & Group 2 & 219.5561 & 65.1054 & 36.2118 & 27.7099 & 14.8522 \\
\hline & Group 3 & 220.7921 & 64.7638 & 36.4818 & 27.0298 & 15.3634 \\
\hline & Group 4 & 221.6588 & 65.6042 & 36.1307 & 28.3611 & 15.5129 \\
\hline & Group 5 & 218.1416 & 66.5646 & 38.8615 & 28.4011 & 15.9406 \\
\hline \multirow{5}{*}{ Severe wear } & Group 1 & 253.2741 & 88.1292 & 40.9097 & 27.4871 & 15.9595 \\
\hline & Group 2 & 255.3187 & 89.2155 & 37.3920 & 25.3073 & 16.6829 \\
\hline & Group 3 & 228.0774 & 92.6607 & 43.8101 & 25.8627 & 17.3696 \\
\hline & Group 4 & 245.3930 & 98.4203 & 39.6401 & 24.2324 & 17.5652 \\
\hline & Group 5 & 253.1356 & 96.0376 & 38.6695 & 25.4074 & 15.6127 \\
\hline
\end{tabular}

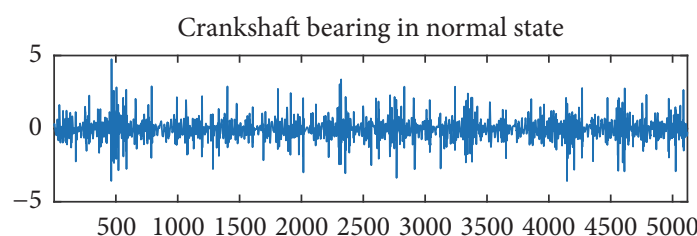

500100015002000250030003500400045005000
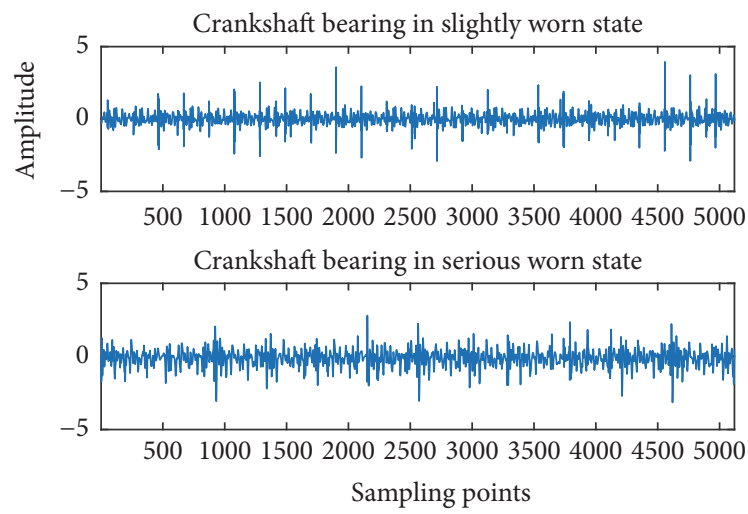

FIGURE 3: Time-domain signal of crankshaft bearing.

crankshaft bearing is shown in Table $2\left(\delta_{1}-\delta_{5}\right.$ are singular values).

The singular values of different IMF components reflect the features of each frequency band of the vibration signal. They also correspond to the energy changes in different frequency band signals [10]. From Table 2, it is found that the singular value matrix changes correspondingly with the wear degree of crankshaft bearings; the singular value of the components in slightly worn state and severely worn state will be higher than those in the normal state; however, it does not increase linearly with the wear degree.

5.2. Fault Identification. In this experiment, the input data dimension of fuzzy neural network is 5 while the output data dimension is 15 . According to the number of input and output nodes of the network, the number of selected membership functions is better to be 10 after the optimization. Therefore, the construction of the network structure is 5-10-15. Table 2 shows input training samples and Table 3 shows testing samples.

Through the operation of fuzzy neural network, the results can be obtained in Table 4 .

In the training process, we trained the normal state of the crankshaft bearings to the value of 1 , the slightly worn state to the value of 2 , and the severely worn state to the value of 3 , as shown in the output trained sample $y$. Combined with the test results, we define the numerical values as the independent variable $x$, and the bearings' state is the function $g(x)$. We can give the decision function as follows:

$$
g(x)= \begin{cases}\text { normal state } & x \leq 1.5 \\ \text { slight wear } & 1.5<x \leq 2.5 \\ \text { severe wear } & x>2.5 .\end{cases}
$$

The decision function can be regarded as the criterion for running state of crankshaft bearings. We can also consider that the closer the value is to the critical one, the more likely the bearing is to be in the corresponding state; that is to say, the running state of crankshaft bearings is closer to the critical state. When the independent variable $x$ is greater than 2.5, this means the bearing should be repaired or replaced in time.

The verification between the output results and the above identification criterion is shown in Figure 5. As can be seen from Figure 5, the numerical indexes can classify the running state of the crankshaft bearing accurately. Therefore, the diagnosis and recognition in the form of numerical value are realized. The accuracy and feasibility of this method can be fully proved by the above experimental results.

\section{Conclusion}

A fault diagnosis method based on EMD-SVD and fuzzy neural network was presented and evaluated. In this paper, 
TABle 3: Detection sample.

\begin{tabular}{|c|c|c|c|c|c|c|}
\hline Wear degree of crankshaft bearing & Signal & $\delta_{1}$ & $\delta_{2}$ & $\delta_{3}$ & $\delta_{4}$ & $\delta_{5}$ \\
\hline \multirow{3}{*}{ Normal state } & Group 1 & 106.6085 & 78.1076 & 51.9938 & 21.4124 & 13.9582 \\
\hline & Group 2 & 104.2226 & 79.5154 & 54.2490 & 23.5038 & 12.2369 \\
\hline & Group 3 & 102.3797 & 75.3013 & 54.9141 & 26.9316 & 14.4099 \\
\hline \multirow{3}{*}{ Slight wear } & Group 1 & 217.1926 & 59.1240 & 36.0639 & 27.7408 & 16.1523 \\
\hline & Group 2 & 215.0976 & 63.3503 & 37.8884 & 28.2991 & 19.0441 \\
\hline & Group 3 & 217.9721 & 64.7321 & 37.0377 & 27.6948 & 15.9726 \\
\hline \multirow{3}{*}{ Severe wear } & Group 1 & 246.6129 & 97.0573 & 39.1976 & 27.3352 & 17.0874 \\
\hline & Group 2 & 242.2781 & 103.4151 & 41.3402 & 27.5708 & 17.8415 \\
\hline & Group 3 & 253.2741 & 88.1292 & 40.9097 & 27.4871 & 15.9595 \\
\hline
\end{tabular}

TABLE 4: Results of the operation of fuzzy neural network.

\begin{tabular}{lrr}
\hline Calculation error & Output trained sample & The result of fault feature recognition \\
\hline & $y=[1.00001 .00001 .00001 .00001 .0000$ & $y t=[1.06751 .15131 .0000$ \\
$7.14 E-09$ & 1.99941 .99902 .00072 .00061 .9999 & 1.80922 .07081 .9994 \\
& $2.99992 .99993 .00032 .99993 .0000]$ & $3.08993 .27222 .9999]$ \\
\hline
\end{tabular}
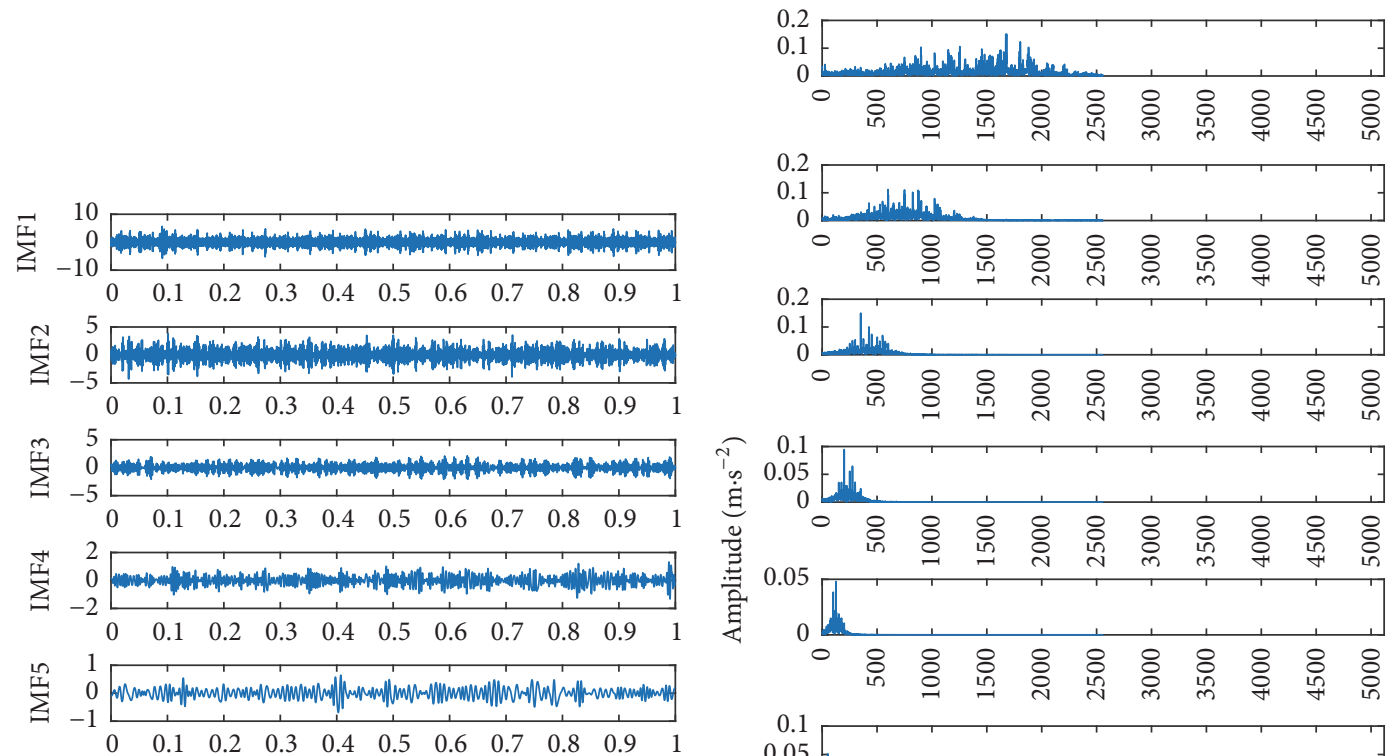

$\sum_{-0.5}^{0} \begin{array}{lllllllllll}0.5 \\ 0\end{array}$

$\sum_{-0.5}^{0.5}$

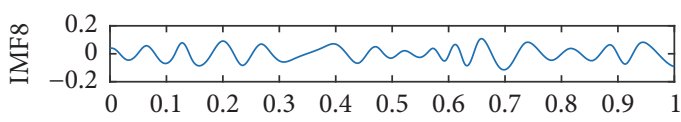

$t(\mathrm{~s})$

(a)
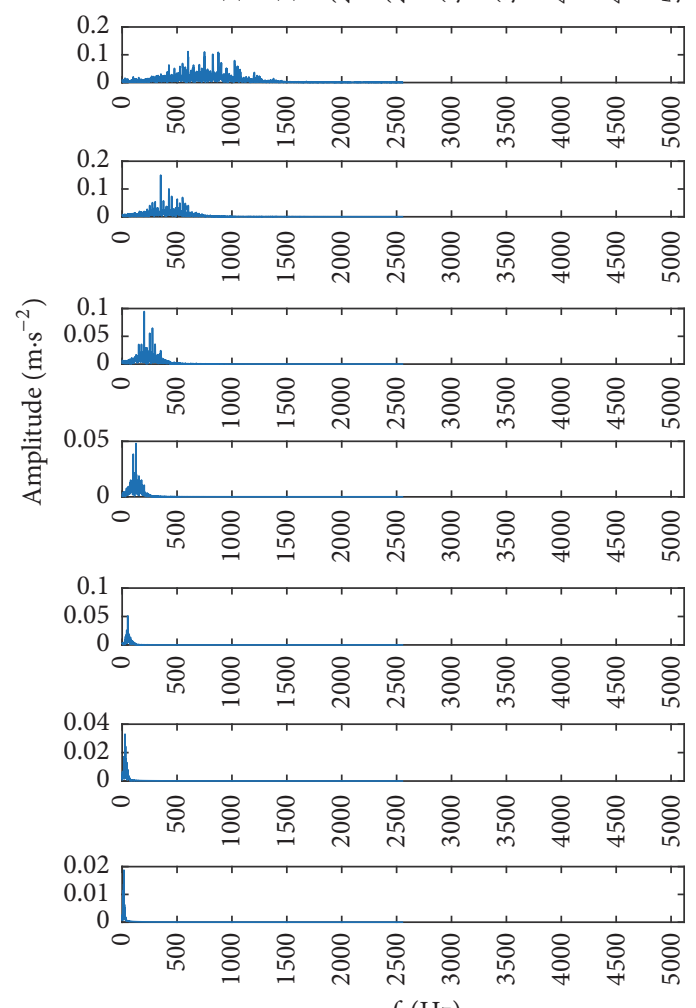

$f(\mathrm{~Hz})$

(b)

FIGURE 4: The first 8 IMFS and their frequency-domain diagrams after EMD of crankshaft bearing vibration signal in normal state. 


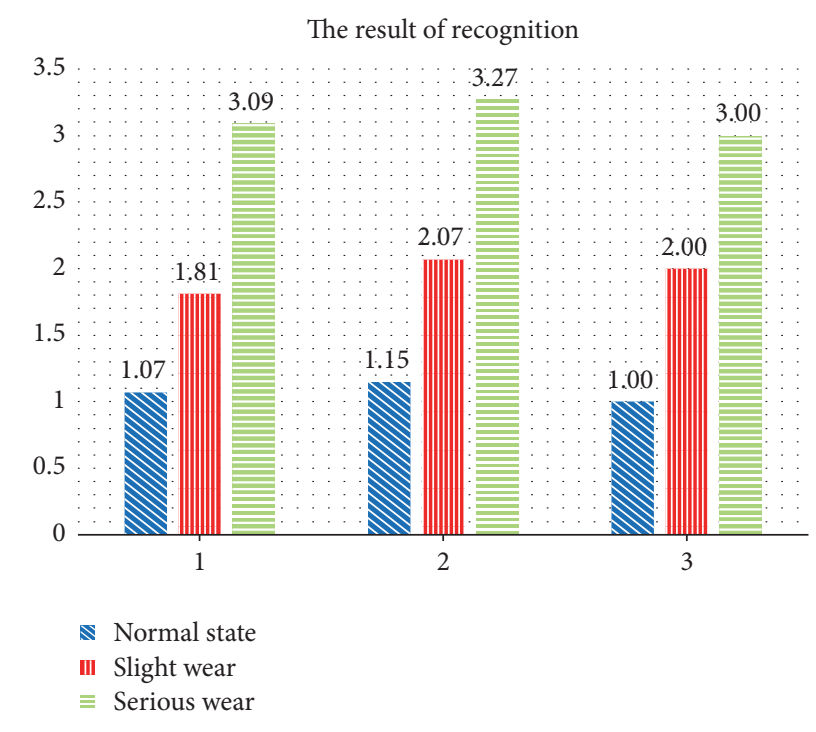

FIGURE 5: Comparison of output results and training standards.

empirical mode decomposition was optimized by singular value decomposition, and the singular value was used as the training sample of the fuzzy neural network. The diagnosis and recognition in the form of numerical values can be realized. Through the combination of EMD-SVD and fuzzy neural network, the following conclusions can be obtained according to the experimental analysis.

(1) SVD can reduce the noise components and intermittent signals. Because the singular value has high stability, it can realize the optimization of EMD. Therefore, the fault features extracted by the combination of EMD and SVD have higher accuracy, which lays a good foundation for the identification of fault states.

(2) The experimental results showed that the singular value can reflect the features of each frequency band of the vibration signal, which corresponds to the energy changes of signals in different frequency bands.

(3) The fuzzy neural network not only can identify the fault features by numerical value with high recognition accuracy but also has a positive correlation with the gradual change of fault state.

(4) The combination of EMD-SVD and fuzzy neural network can effectively compensate the shortcomings of the previous research, and it is of great significance to the fault diagnosis of crankshaft bearings.

\section{Data Availability}

The data used to support the findings of this study are available from the corresponding author upon request.

\section{Conflicts of Interest}

The authors declare no conflicts of interest.

\section{Authors' Contributions}

Jingbo Gai provided the main idea of the study, and Yifan $\mathrm{Hu}$ analyzed the experiment and completed the paper.

\section{References}

[1] J. B. Ali, N. Fnaiech, L. Saidi, B. Chebel-Morello, and F. Fnaiech, "Application of empirical mode decomposition and artificial neural network for automatic bearing fault diagnosis based on vibration signals," Applied Acoustics, vol. 89, pp. 16-27, 2015.

[2] Y. Yang et al., "Rolling bearing fault diagnosis method based on neural network," Journal of Vibration and Shock, vol. 24, no. 1, pp. 87-90, 2005.

[3] T. Xia, X. Wang, Y. Xiao et al., "Application of EMD-AR to extract diesel engine crankshaft bearing fault characteristics," Journal of Vibration, Test and Diagnosis, vol. 30, no. 03, pp. 318321, 2010.

[4] J. Si, J. Ma, J. Niu, and E. Wang, "An intelligent fault diagnosis expert system based on fuzzy neural network," Journal of Vibration and Shock, vol. 36, no. 4, pp. 164-171, 2017.

[5] L. Zhang et al., "Fault diagnosis of engine crankshaft bearing based on EEMD and Fuzzy C-means clustering algorithm," Journal of Combustion Engines, vol. 29, no. 4, pp. 332-336, 2011.

[6] M. Guo, L. Xu, X. Miao et al., "A method for extracting vibration signal characteristics of distribution switch using singular value decomposition of time-frequency matrix," Chinese Society for Electrical Engineering, vol. 34, no. 28, pp. 4990-4997, 2014.

[7] C. Zhou, X. Wu, C. Liu et al., "Fault diagnosis of rolling bearing based on EMD and fuzzy c-means clustering," Journal of Kunming University of Science and Technology (Science and Technology), vol. 34, no. 06, pp. 34-39, 2009.

[8] T. Guo, Z. Deng, and M. Xu, "Application of improved EMD algorithm based on particle swarm optimization in bearing fault feature extraction," Journal of Vibration and Shock, vol. 36, no. 16, pp. 182-187, 2017.

[9] J. D. Zheng, J. S. Cheng, and Y. Yang, "Generalized empirical mode decomposition and its applications to rolling element bearing fault diagnosis," Mechanical Systems and Signal Processing, vol. 40, no. 1, pp. 136-153, 2013.

[10] L. Zhang and J. Xiao, Machine Fault Diagnosis Technology Based on MATLAB Case Tutorial, Higher Education Press, Beijing, China, 2016.

[11] A. Soualhi, K. Medjaher, and N. Zerhouni, "Bearing health monitoring based on hilbert-huang transform, support vector machine, and regression," IEEE Transactions on Instrumentation and Measurement, vol. 64, no. 1, pp. 52-62, 2015.

[12] J. Dybała and R. Zimroz, "Rolling bearing diagnosing method based on empirical mode decomposition of machine vibration signal," Applied Acoustics, vol. 77, pp. 195-203, 2014. 


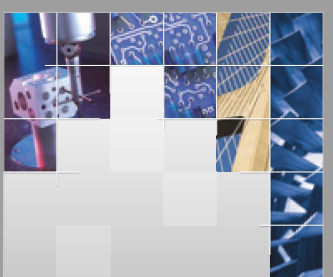

\section{Enfincering}
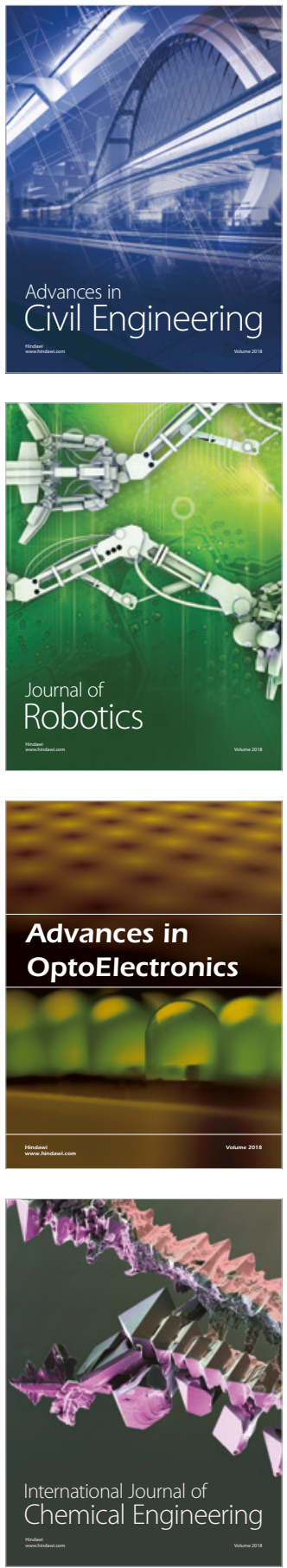

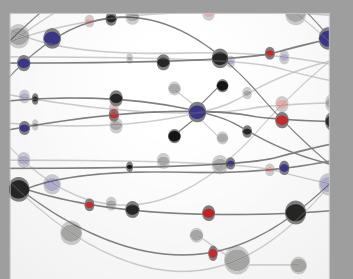

\section{Rotating \\ Machinery}

The Scientific World Journal

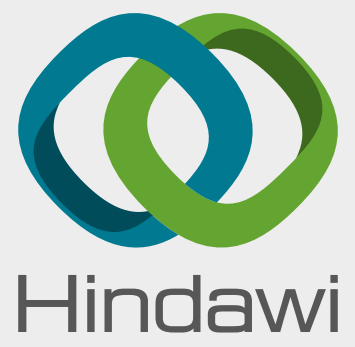

Submit your manuscripts at

www.hindawi.com
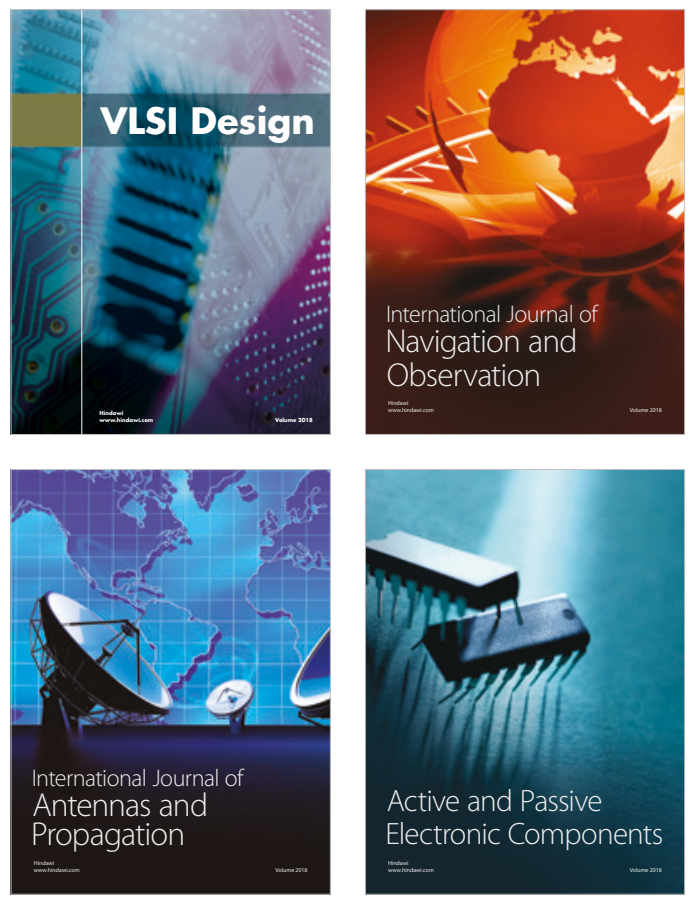
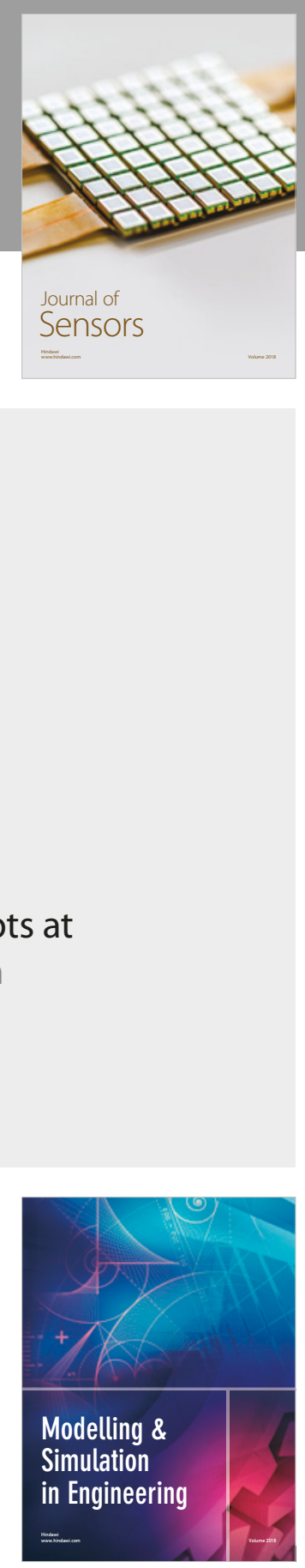

\section{Advances \\ Multimedia}
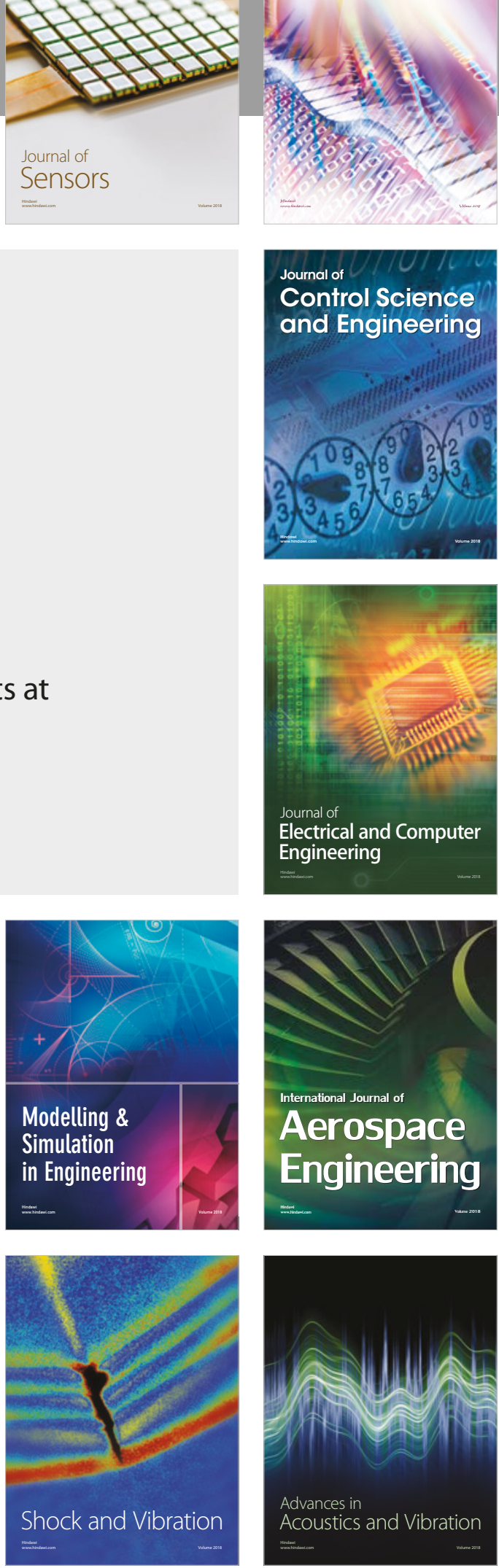